\title{
Educating non-cardiologists improves the initial management of patients with non-ST elevated myocardial infarction
}

\author{
Authors: Rebecca Clifford, ${ }^{\mathrm{A}}$ Ramesh Nadarajah, ${ }^{\mathrm{A}}$ Shankar Patil, ${ }^{\mathrm{A}}$ Peysh Patel ${ }^{\mathrm{B}}$ and Sanjay Gupta ${ }^{\mathrm{A}}$
}

\section{Introduction}

European Society of Cardiology (ESC) guidelines recommend treating patients presenting with suspected non-ST elevation myocardial infarction (NSTEMI) with aspirin, ticagrelor and fondaparinux in the absence of contraindications. ${ }^{1}$ However, most admissions are via the emergency department (ED) or acute assessment unit (AAU) where the initial consultation is with a noncardiologist. We speculated whether this resulted in poor adherence with guideline-directed treatment and potential adverse outcomes.

\section{Purpose}

To assess if patients diagnosed with NSTEMI in the AAU and ED are treated as per ESC guidelines. To improve the implementation of guideline-directed therapy by education.

\section{Methods}

A retrospective review of consecutive patients referred to the cardiology unit with NSTEMI was conducted over a 6-week period at a busy district hospital (Group 1). Data was collated on baseline demographics, fulfilment of diagnostic criteria, pharmacotherapy and origin of referral. Cases were additionally interrogated for adverse outcomes, including thrombolysis in myocardial infarction (TIMI) bleed and cardiovascular mortality. A subsequent intervention was conducted with the creation and distribution of a poster accompanied by outreach teaching sessions to physicians and advanced nurse practitioners (ANPs). A repeat prospective study was carried out using similar methodology (Group 2). Differences between groups were analysed using a two-tailed t-test or chi-squared test, with $\mathrm{p}$-values $<0.05$ considered statistically significant.

\section{Results}

There were 66 patients in Group 1 and 60 patients in Group 2. There were no significant differences in age or gender. ESC criteria for NSTEMI were met in $27 \%$ in Group $1(18 / 66)$ and $28 \%$ in Group 2 (17/60). Among NSTEMI patients not already on an anticoagulant the intervention produced an improvement in the number of patients treated with aspirin, ticagrelor and fondaparinux (Group 1: 4/11 (36\%), Group 2: 10/12 (83\%), $\mathrm{p}=0.06$ ). In addition, while previously not a single NSTEMI patient on anticoagulation was treated with the addition of antiplatelet therapy (Group 1: 0/7 (0\%)), post-intervention this improved (Group $2: 4 / 6,(66 \%))$ and met statistical significance $(p=0.006)$. Most worryingly, one patient in Group 1 on anticoagulation received loading with each of aspirin, ticagrelor, clopidogrel, fondaparinux and apixaban. There were no deaths or TIMI major or minor bleeds in either group.

\section{Conclusions}

In this small, single centre study, targeted education produced a significant increase in the number of patients receiving appropriate NSTEMI treatment. This was especially the case in patients who were admitted with a NSTEMI on anticoagulants, a group that is theoretically at the highest risk of adverse bleeding events.

\section{Reference}

1 European Society of Cardiology. 2015 ESC Guidelines for the management of acute coronary syndromes in patients presenting without persistent ST-segment elevation. Eur Heart J 2016;37:267315.

Authors: ${ }^{\text {AY }}$ ork Hospital; ${ }^{\text {P Pinderfields Hospital }}$ 